\title{
Prevalence and characteristics of pregnancy and lactation related calls to the National Poison Centre in Belgium: a retrospective analysis of calls from 2012 to 2017
}

This abstract is intended for: Free communication (15 min)

Michael Ceulemans (PhD researcher, Department of Pharmaceutical and Pharmacological Sciences, KU Leuven, Belgium; e-mail: michael.ceulemans@kuleuven.be)

Marijke Fortuin (National Poison Centre Belgium; e-mail: marijke.fortuin@ poisoncentre.be)

Kristel Van Calsteren (Professor, Obstetrics \& Gynecology, University Hospital Leuven \& Department of Development and Regeneration, KU Leuven, Belgium; e-mail: kristel.vancalsteren@uzleuven.be)

Karel Allegaert (Professor, Department of Development and Regeneration, KU Leuven, Belgium \& Department of Pediatrics, Erasmus MC, Rotterdam, The Netherlands; e-mail: karel.allegaert@kuleuven.be)

Veerle Foulon (Professor, Department of Pharmaceutical and Pharmacological Sciences, KU Leuven, Belgium; e-mail : veerle.foulon@kuleuven.be

\section{INTRODUCTION}

In the absence of a teratology information service in Belgium, the national Poison Centre might act as a substitute centre for answering pregnancy and lactation related questions regarding medication use. The aim of this study was to define the prevalence and characteristics of pregnancy and lactation related calls to the national Poison Centre in Belgium, as well as the type of health products involved during these calls.

\section{METHODS}

A retrospective, descriptive study on pregnancy and lactation related calls involving health products received by the Belgian Poison Centre between January 2012 and December 2017 was performed.

\section{RESULTS}

The Poison Centre annually received about 361 calls related to pregnancy and lactation. Pregnant and lactating women mainly called the Poison Centre themselves in case of exposure, while relatives were the predominant type of caller when preventive information was requested. The Poison Centre was mostly contacted for information about medicines, and especially for preventive questions during lactation. Many questions involved over-the-counter medicines such as paracetamol and ibuprofen. Given the safety issues related to some involved products (ibuprofen, zolpidem, benzodiazepines and pseudoephedrine), seeking for advice was justified.

\section{CONCLUSIONS}


The Belgian Poison Centre received almost daily calls on medication exposure during pregnancy and lactation. These findings contribute to the ongoing discussion to establish a teratology information service in Belgium. 